\title{
Differential expression profile analysis of cisplatin-regulated miRNAs in a human gastric cancer cell line
}

\author{
CHUNLIN YIN ${ }^{1}$, XIANXIAN ZHENG ${ }^{2}$, HEPING XIANG $^{3}$, HE $^{3}{ }^{3}$, MING GAO $^{3}$, \\ XIANGLING MENG ${ }^{1}$ and KAI YANG ${ }^{4}$ \\ ${ }^{1}$ Department of General Surgery, The First Affiliated Hospital of Anhui Medical University, Hefei, Anhui 230022; \\ ${ }^{2}$ Department of Clinical Laboratory, Hefei Binhu Hospital, Hefei, Anhui 230601; ${ }^{3}$ Department of Emergency Surgery; \\ ${ }^{4}$ Department of Clinical Laboratory, The Second Affiliated Hospital of Anhui Medical University, \\ Hefei, Anhui 230032, P.R. China
}

Received August 14, 2018; Accepted May 22, 2019

DOI: $10.3892 / \mathrm{mmr} .2019 .10430$

\begin{abstract}
Cisplatin, one of the most commonly used drugs in combination chemotherapy, is an effective anti-tumor agent widely used for diverse tumor types. MicroRNAs (miRNAs/miRs) are involved in the occurrence, development, diagnosis and treatment of cancer. Therefore, the aim of the current study was to explore whether cisplatin exerts anticancer effects by causing differential expression of miRNAs in human gastric cancer cells. The human gastric cancer cell line NCI-N87 was cultured with a certain dose of cisplatin and high-throughput sequencing combined with reverse transcription-quantitative polymerase chain reaction (RT-qPCR) was performed to detect cisplatin-regulated miRNAs. miRNAs upregulated and downregulated following cisplatin exposure were analyzed. High-throughput sequencing revealed 33 upregulated and 16 downregulated miRNAs. A total of five significantly upregulated and five significantly downregulated miRNAs were identified by RT-qPCR. The expression levels of hsa-miR-1246 and hsa-miR-892b were consistent with the results obtained from high-throughput sequencing. Gene Ontology and Kyoto Encyclopedia of Genes and Genomes pathway clustering of cisplatin-regulated miRNAs revealed that the miRNAs regulated genes involved in several biological processes and signaling pathways. The results obtained in the current study suggested that cisplatin may exert an important anticancer effect in gastric cancer via complex biological processes and signaling pathways.
\end{abstract}

Correspondence to: Dr Xiangling Meng, Department of General Surgery, The First Affiliated Hospital of Anhui Medical University, 218 Jixi Road, Hefei, Anhui 230022, P.R. China

E-mail: xianglingmeng55@sina.cn

Key words: cisplatin, gastric cancer, microRNA, high-throughput sequencing, differential expression

\section{Introduction}

Cisplatin (cis-dichlorodiammine platinum) is a platinum-based chemotherapeutic agent used for the treatment of several types of cancer. It binds to DNA and inhibits DNA replication, resulting in apoptosis $(1,2)$. Cisplatin has a strong anti-cancer effect and synergistic action with various anti-tumor drugs and is therefore one of the most commonly used drugs in combination chemotherapy $(3,4)$. It is primarily used in the treatment of ovarian, prostate, testicular, lung and gastric cancer $(3,4)$. Cisplatin is associated with dose-dependent side effects $(5,6)$, including gastrointestinal reactions (7), bone marrow suppression (8), liver and kidney damage (9), ototoxicity (10) and neurotoxicity (11). Renal toxicity is frequently encountered, with a clinical incidence rate of $25-35 \%$ (12-14). The side effects associated with cisplatin have limited its use and clinical efficacy $(15,16)$. Therefore, strategies to reduce these side effects are required in the field of antitumor therapy.

Gastric cancer, which is a malignant tumor originating from the gastric mucosal epithelium (17), is the fourth most common cancer and the second most common cause of cancer-associated mortality worldwide (18). According to global cancer statistics in 2012, gastric cancer accounted for approximately one million new cases and over 700,000 mortalities, representing $8 \%$ of all cancer cases and $9.7 \%$ of all cancer mortalities, and almost half of these patients came from China (19). Although the morbidity and mortality of gastric cancer has decreased in recent years, the 5-year survival rate remains low $(<30 \%)(20,21)$. Radiotherapy and chemotherapy combined with surgical resection is the main treatment for gastric cancer, but the recurrence rate is very high $(20,21)$. Cisplatin is currently a first line chemotherapeutic agent for gastric cancer $(22,23)$, particularly for patients with advanced gastric cancer.

Cisplatin has a better curative effect for gastric cancer. The target microRNAs (miRNAs) of cisplatin remain unknown. In the current study, the human gastric cancer cell line NCI-N87 was used to identify differentially expressed miRNAs following exposure to cisplatin. High-throughput sequencing and bioinformatics analysis identified 49 differentially expressed miRNAs, including 33 upregulated miRNAs 
and 16 downregulated miRNAs following treatment with cisplatin. Reverse-transcription quantitative polymerase chain reaction (RT-qPCR) results revealed that the expression level of hsa-miR-1246 and hsa-miR-892b were consistent with the microarray data. The results obtained in the current study suggested that cisplatin may serve a role in the treatment of gastric cancer by regulating the expression of the aforementioned miRNAs, which may allow the development of novel therapeutic agents for gastric cancer.

\section{Materials and methods}

Cell culture and cisplatin stimulation. The human gastric cancer cell line NCI-N87 (American Type Culture Collection) was cultured in Dulbecco's Modified Eagles Medium (DMEM; HyClone; GE Healthcare Life Sciences) supplemented with $10 \%$ fetal bovine serum (HyClone; GE Healthcare Life Sciences) and $1 \%$ penicillin and streptomycin (Suzhou Zeke Biotech, Co., Ltd.). Cells were maintained in an incubator at $5 \% \mathrm{CO}_{2}$ and $37^{\circ} \mathrm{C}$. When the cell confluence reached $70-80 \%$, the cells were treated $0,7.5,15,30$ and $60 \mu \mathrm{g} / \mathrm{ml}$ cisplatin for $48 \mathrm{~h}$ at $37^{\circ} \mathrm{C}$. Cell viability was subsequently assessed using an MTT assay and applied for further investigation.

MTT assay. A total of $1 \times 10^{4}$ NCI-N87 cells were seeded in 96-well plates with complete DMEM and incubated at $37^{\circ} \mathrm{C}$ for 48 h. Cells were treated with MTT (Changchun Keygen Biological Products Co., Ltd.) according to the manufacturer's instructions and incubated in an incubator at $5 \% \mathrm{CO}_{2}$ and $37^{\circ} \mathrm{C}$ for $4 \mathrm{~h}$. The purple formazan crystals were subsequently dissolved in $150 \mu \mathrm{l}$ dimethyl sulfoxide (Sigma-Aldrich; Merck $\mathrm{KGaA}$ ) at room temperature for $10 \mathrm{~min}$, and analyzed at a wavelength of $490 \mathrm{~nm}$ using a microplate reader.

High-throughput sequencing. The total RNA of NCI-N87 cells treated with and without cisplatin was extracted using the Takara MiniBEST Universal RNA Extraction Kit (cat. no. 9767; Takara Bio, Inc.) according to the manufacturer's protocol and sent to Shanghai Personal Biotechnology Co., Ltd. for high-throughput sequencing using the Illumina NextSeq500 platform (Illumina, Inc.).

RT-qPCR assay. The total RNA was extracted using the Takara MiniBEST Universal RNA Extraction Kit (cat. no. 9767; Takara Bio, Inc.). Total RNA was reverse transcribed and qPCR was performed using a PrimeScript One Step RT-PCR Kit Ver.2 (cat. no. RR057A; Takara Bio, Inc.) according to the manufacturers' instructions. The reaction mixture, which consisted of $2 \mu 1$ 5X PrimeScript RT Master mix (included in the PrimeScript One Step RT-PCR Kit), 500 ng total RNA, RNase-free $\mathrm{dH}_{2} \mathrm{O}$ up to $10 \mu \mathrm{l}$, was prepared and reacted at $37^{\circ} \mathrm{C}$ for $15 \mathrm{~min}$, followed by $85^{\circ} \mathrm{C}$ for $5 \mathrm{sec}$ and $4^{\circ} \mathrm{C}$ indefinitely. qPCR was subsequently performed using the following mixture: $10 \mu 1$ of 2x SYBR Premix Ex Taq II (included in the PrimeScript One Step RT-PCR Kit), $0.8 \mu 1$ of forward primer, $0.8 \mu \mathrm{l}$ of reverse primer, $0.4 \mu \mathrm{l}$ of ROX Reference Dye II (included in the kit), $2 \mu \mathrm{l}$ of cDNA and $6 \mu \mathrm{l}$ of $\mathrm{ddH}_{2} \mathrm{O}$. U6 was used as reference gene. The thermocycling conditions were as follows: One cycle of $95^{\circ} \mathrm{C}$ for $30 \mathrm{sec}, 40$ cycles of $95^{\circ} \mathrm{C}$ for $5 \mathrm{sec}, 60^{\circ} \mathrm{C}$ for $34 \mathrm{sec}$ and $4^{\circ} \mathrm{C}$ indefinitely. The data was analyzed using SDS software (version 1.4; Applied Biosystems; Thermo Fisher Scientific, Inc.) based on the $2^{-\Delta \Delta \mathrm{Cq}}$ method $(24,25)$, and histogram analysis was performed using Origin software (version 10.5.30; https://www.originlab. com/index.aspx?go=PRODUCTS/Origin). All primers are listed in Table I.

Bioinformatics analysis of differentially expressed miRNAs in NCI-N87 cells following cisplatin treatment. High-throughput sequencing was performed with $>3$ biological replicates in each group, and t-tests were used to select differentially expressed genes using fold change (FC). FC differences in the cisplatin-treated ( 3 replicates) and control (3 replicates) groups were investigated. $\mathrm{P}<0.05$ and $\log 2$ (FC) $>2$ (upregulated) or $\log 2(\mathrm{FC})<-2$ (downregulated) were used to identify differentially expressed miRNAs. The differentially expressed miRNAs were used to construct heatmaps and perform volcano analysis using $\mathrm{R}$ (version 3.5, www.R-project.org). The distribution frequency of the miRNAs following cisplatin treatment was determined by the adjusted P-value (Padj) according to the analysis of differentially expressed miRNAs (26).

Gene Ontology (GO) clustering. The GO database (geneontology.org) contains three aspects of functional information: The biological process (BP), the cellular component (CC), and the molecular function (MF) (27,28). These functions were organized into the 'Directed Acyclic Graph' (DAG) structure according to the size of the concept. Genes are considered significantly enriched based on the ratio of the observed GO term for all genes/GO term for a single gene set. In the current study, each gene annotated to a GO term was extensively annotated to all parent nodes of that node. Each GO term-enriched $\mathrm{P}$-value was calculated using the hyper-geometric distribution method, and the P-value was corrected using the false discovery rate. $\mathrm{P}<0.05$ was considered to indicate a statistically significant difference. Redundant GO terms within the threshold were removed; GO terms terminal to the DAG graph which were significantly enriched were selected.

Kyoto Encyclopedia of Genes and Genomes (KEGG) pathway clustering. The KEGG database (https://www.genome. $\mathrm{jp} / \mathrm{kegg} /$ ) is the world's largest database for analyzing gene function and genomic information from a biological pathway perspective system (29). It contains metabolites, enzymes, biochemical reactions, gene regulation and protein interactions. In the current study, the hypergeometric distribution method was used to study biological pathways. The F-value was used to correct the P-value and the threshold was set to 0.05 . Finally, a KEGG signaling pathway in which differentially expressed genes were significantly enriched was determined. The network was analyzed using the Cytoscape software (version 3.7.1; https://cytoscape.org/download.html).

Target prediction. miR-1246-regulated genes were obtained using TargetScan (version 7.2; http://www.targetscan. org/vert_72/) (30) and the miRDB database (http://www. mirdb.org/) (31).

Statistical analysis. All data were expressed as mean \pm standard deviation. One-way ANOVA followed by the Least Significant 
Table I. Primers used for the reverse-transcription polymerase chain reaction.

\begin{tabular}{|c|c|}
\hline Primer & Sequence $\left(5^{\prime} \rightarrow 3^{\prime}\right)$ \\
\hline hsa-miR-3609-F & 5'-ACACTCCAGCTGGGCAAAGTGATGAGTAATAC-3' \\
\hline hsa-miR-3609-R & 5'-CTCAACTGGTGTCGTGGAGTCGGCAATTCAGTTGAGCAGCCAGT-3' \\
\hline hsa-miR-4497-F & 5'-ACACTCCAGCTGGGCTCCGGGACGG-3' \\
\hline hsa-miR-4497-R & 5'-CTCAACTGGTGTCGTGGAGTCGGCAATTCAGTTGAGGCCCAGCC-3' \\
\hline hsa-miR-1246-F & 5'-ACACTCCAGCTGGGAATGGATTTTTGG-3' \\
\hline hsa-miR-1246-R & 5'-CTCAACTGGTGTCGTGGAGTCGGCAATTCAGTTGAGCCTGCTCC-3' \\
\hline hsa-miR-4301-F & 5'-ACACTCCAGCTGGGTCCCACTACTTCAC-3' \\
\hline hsa-miR-4301-R & 5'-CTCAACTGGTGTCGTGGAGTCGGCAATTCAGTTGAGTCACAAGT-3' \\
\hline hsa-miR-6724-5p-F & 5'-ACACTCCAGCTGGGCTGGGCCCGCGGCGGGC-3' \\
\hline hsa-miR-6724-5p-R & 5'-CTCAACTGGTGTCGTGGAGTCGGCAATTCAGTTGAGCCCCACGC-3' \\
\hline hsa-miR-4508-F & 5'-ACACTCCAGCTGGGGCGGGGCTGGG-3' \\
\hline hsa-miR-4508-R & 5'-CTCAACTGGTGTCGTGGAGTCGGCAATTCAGTTGAGCGCGCGCC-3' \\
\hline hsa-miR-33b-3p-F & 5'-ACACTCCAGCTGGGCAGTGCCTCGGCAGTG-3' \\
\hline hsa-miR-33b-3p-R & 5'-CTCAACTGGTGTCGTGGAGTCGGCAATTCAGTTGAGGGGCTGCA-3' \\
\hline hsa-miR-1268b-F & 5'-ACACTCCAGCTGGGCGGGCGTGGTGGTG-3' \\
\hline hsa-miR-1268b-R & 5'-CTCAACTGGTGTCGTGGAGTCGGCAATTCAGTTGAGCACCCCCA-3' \\
\hline hsa-miR-153-5p-F & 5'-ACACTCCAGCTGGGTCATTTTTGTGATGTT-3' \\
\hline hsa-miR-153-5p-F & 5'-CTCAACTGGTGTCGTGGAGTCGGCAATTCAGTTGAGAGCTGCAA-3' \\
\hline hsa-miR-892b-F & 5'-ACACTCCAGCTGGGCACTGGCTCCTTTCTG-3' \\
\hline hsa-miR-892b-R & 5'-CTCAACTGGTGTCGTGGAGTCGGCAATTCAGTTGAGTCTACCCA-3' \\
\hline U6-F & 5'-CTCGCTTCGGCAGCACA-3' \\
\hline U6-R & 5'-AACGCTTCACGAATTTGCGT-3' \\
\hline
\end{tabular}

Hsa, homo sapiens; miR, microRNA; F, forward; R, reverse.

Difference test was performed. The Student's t-test was used to analyze two groups. Statistical analysis was performed using SPSS software (version 22.0; IBM Corp.). $\mathrm{P}<0.05$ and $\mathrm{P}<0.01$ were considered to indicate significant and highly significant statistical differences, respectively.

\section{Results}

Differential expression profile analysis of cisplatin-regulated miRNAs. According to the quantile normalization exhibited in Fig. 1, the length of the miRNAs identified was 19-25 nucleotides. Bioinformatics analysis revealed a total of 33 upregulated miRNAs and 16 downregulated miRNAs in NCI-N87 cells following cisplatin treatment (Table II). A heatmap was generated based on these differentially expressed miRNAs, where red represented the upregulated miRNAs and green represented the downregulated miRNAs (Fig. 2A). The distribution frequency of the miRNAs following cisplatin treatment was determined by the Padj according to the analysis of differentially expressed miRNAs. The volcano plot presented in Fig. 2B reflected the similarities and the differences in gene expression among the miRNAs regulated by cisplatin treatment.

Expression of hsa-miR-1246 is significantly increased and that expression of hsa-miR-892b is significantly decreased following cisplatin treatment. To further validate the differently expressed miRNAs identified in the microarray,
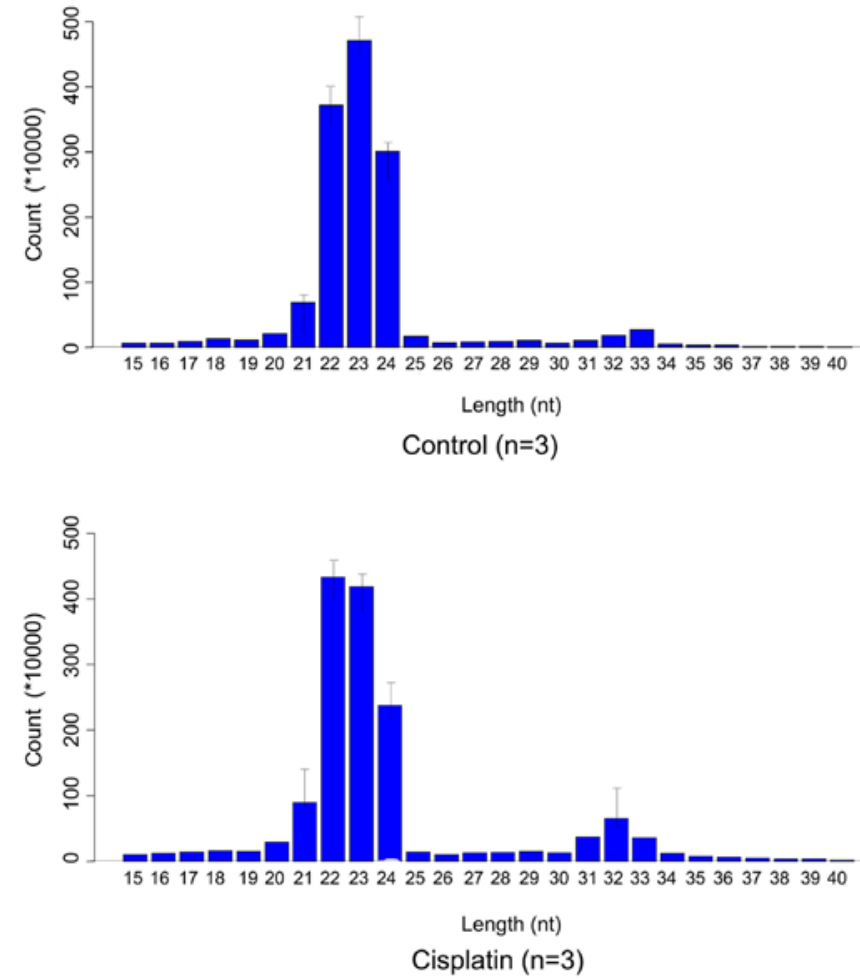

Figure 1. Length distribution of miRNAs sequences. Length distribution of the sequenced miRNAs. Most of sequenced miRNAs exhibited 19-25 nucleotides in length. Hsa, Homo sapiens; miRNA, microRNA. 


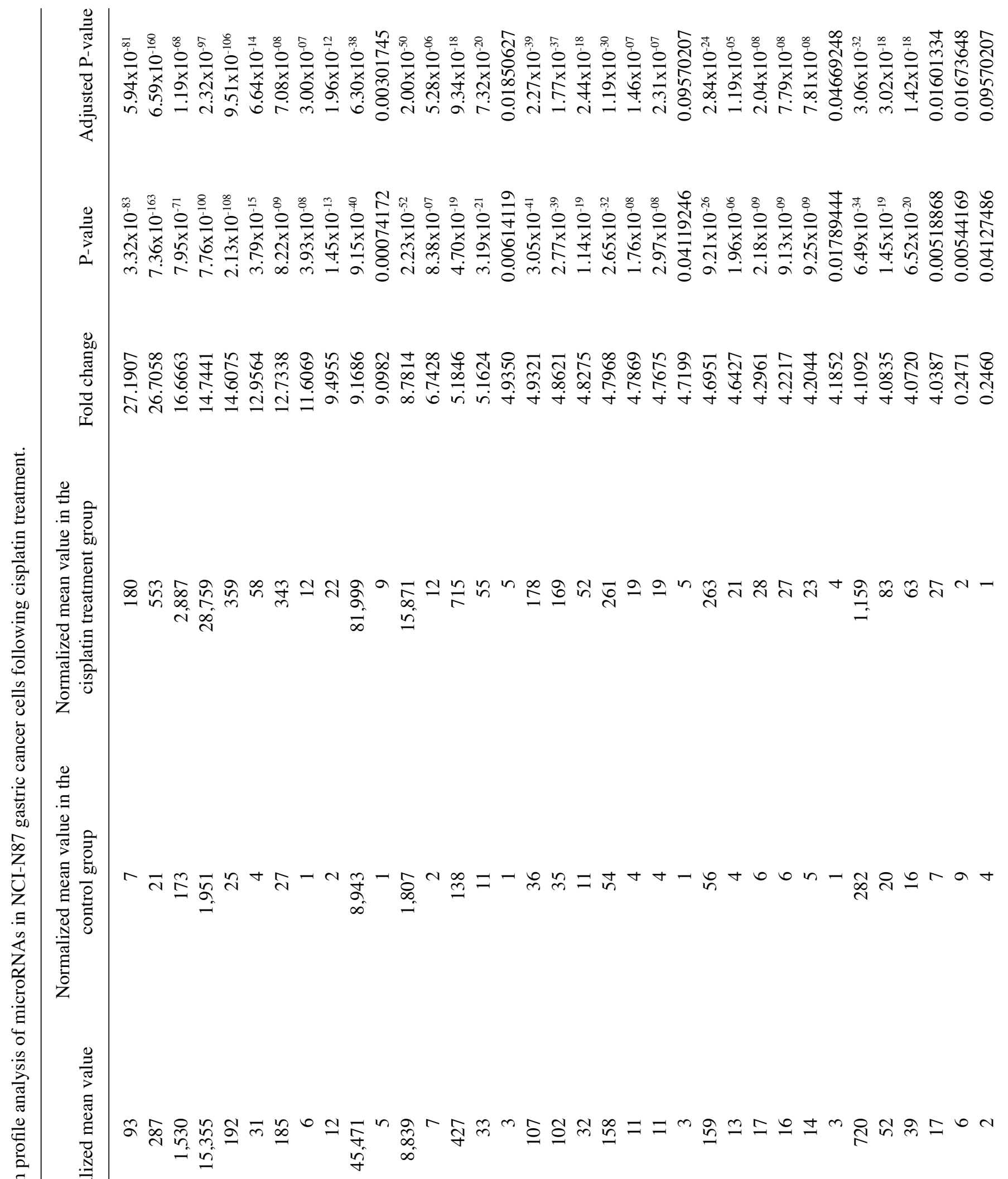

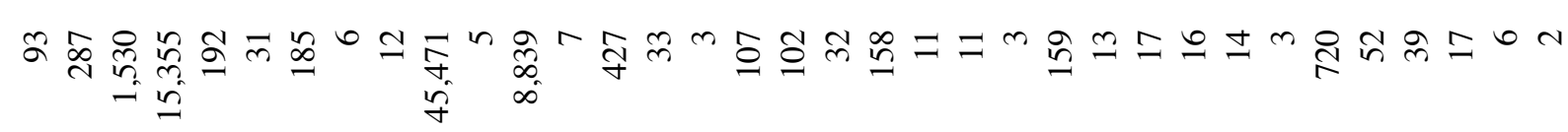

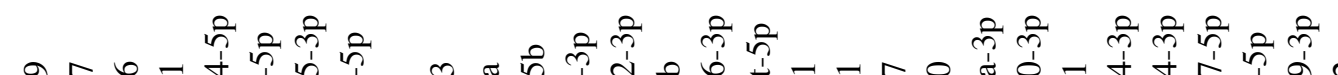

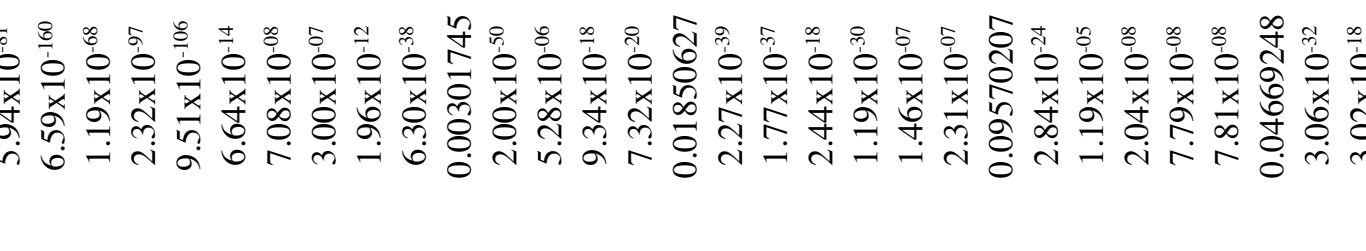




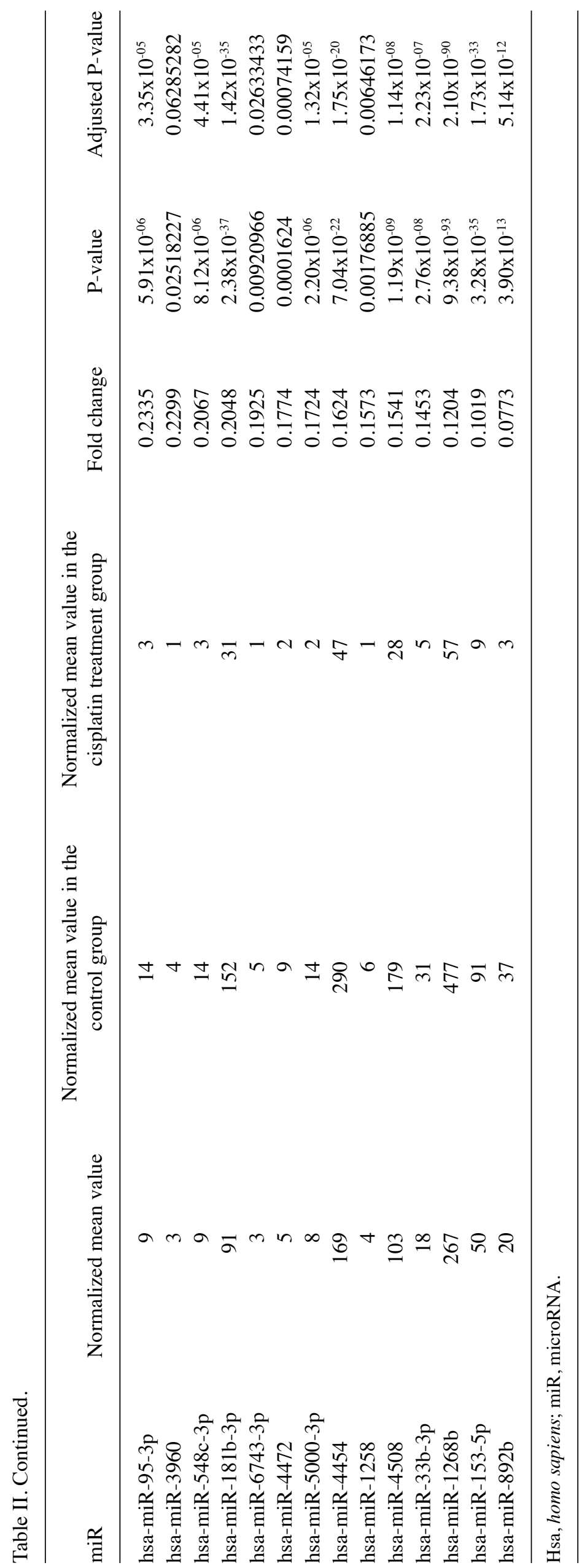



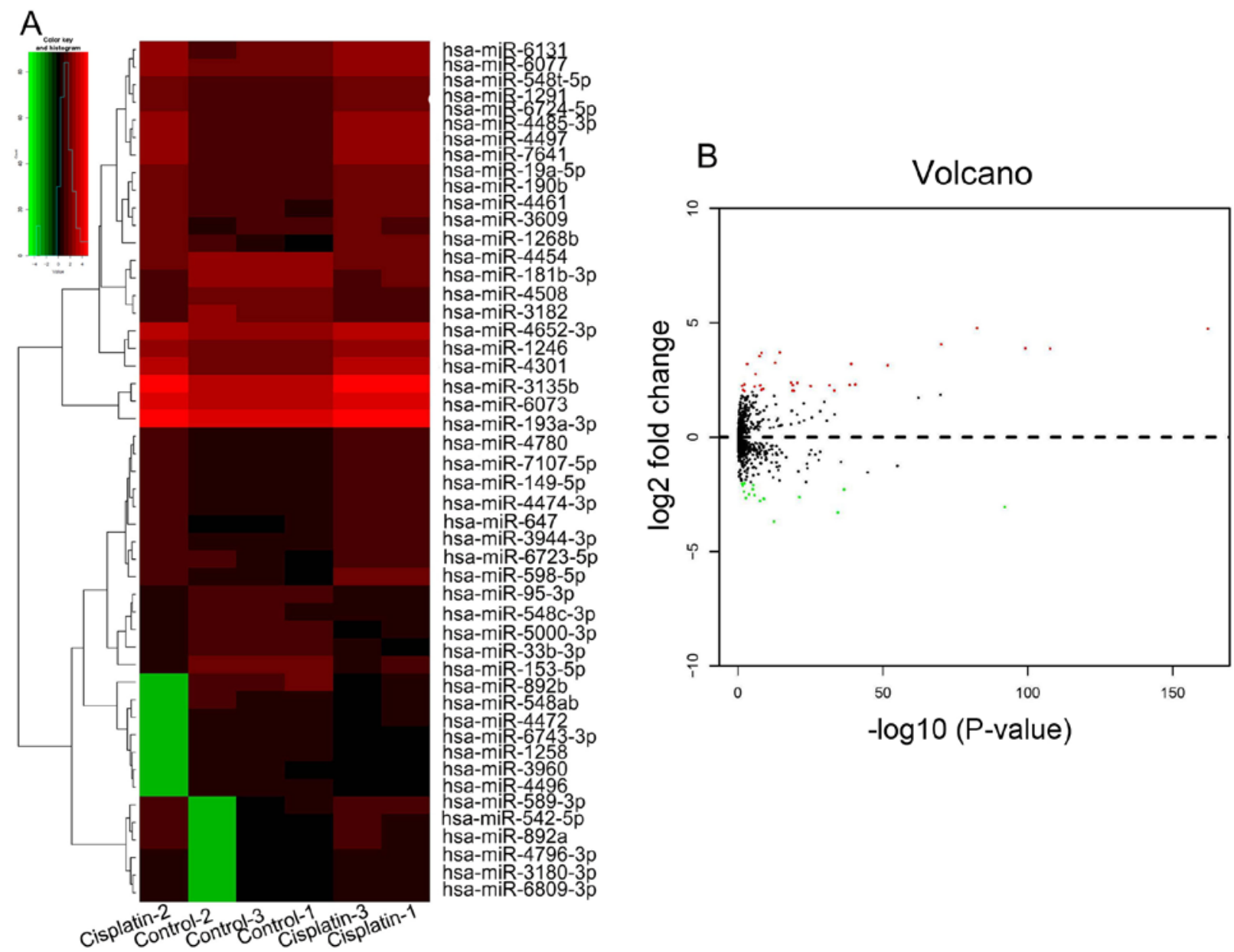

Figure 2. Heatmap and volcano plot analysis of the differentially expressed miRNAs following cisplatin treatment. (A) Heatmap analysis of the differentially expressed miRNAs following cisplatin treatment. Red indicates upregulated miRNAs, and green indicates downregulated miRNAs. The degree of differential expression was indicated by the color intensity. (B) The volcano plot analysis of the differentially expressed miRNAs following cisplatin treatment. The image indicated that with cisplatin treatment, a total of 49 differentially expressed miRNAs were identified, including 33 upregulated and 16 downregulated miRNAs. miRNA, microRNA.

the top five significantly upregulated miRNAs, including hsa-miR-3609, hsa-miR-4497, hsa-miR-1246, hsa-miR-4301 and hsa-miR-6724-5p, and the top five significantly downregulated miRNAs, including hsa-miR-4508, hsa-miR-33b-3p, hsa-miR-1268b, hsa-miR-153-5p and hsa-miR-892b, were selected for RT-qPCR analysis. The expression level of hsa-miR-1246 was significantly increased, and that of hsa-miR-892b was significantly decreased, following treatment of cisplatin compared with the control (Fig. 3), consistent with the results obtained from the microarray. Therefore, hsa-miR-1246 and hsa-miR-892b were selected for subsequent analysis.

GO clustering of hsa-miR-1246 and hsa-miR-892b-regulated genes. For GO analysis, hsa-miR-1246-regulated genes were obtained using TargetScan and the miRDB database. A total of 14 intersecting genes were obtained as presented in the Venn diagram in Fig. 4A. GO clustering was performed to investigate $\mathrm{MF}, \mathrm{BF}$ and $\mathrm{CC}$ associated with the miRs. As presented in Fig. 4B, 10 BPs were identified, including 'transporter activity', 'substrate-specific transporter activity', 'regulation of cellular process', 'regulation of BP', 'protein binding', 'primary metabolic process', 'organic substance metabolic process', 'organic cyclic compound binding', 'organelle' and 'nitrogen compound metabolic process'. Similarly, for hsa-miR-892b regulated genes, a total of 9 intersecting genes was obtained as presented in Venn diagram in Fig. 4C. The GO clustering revealed six processes, including 'regulation of cellular process', 'regulation of biological process', 'protein binding', 'primary metabolic process', 'plasma membrane' and 'organic substance metabolic process' (Fig. 4D). The results obtained indicated that cisplatin-regulated miRNAs participate in a variety of BPs and may have important regulatory roles in the tumorigenesis of gastric cancer.

KEGG pathway clustering of hsa-miR-1246 and hsa-miR-892b regulated genes. As presented in Fig. 5A, the KEGG pathway enrichment based on hsa-miR-1246 regulated genes revealed three pathways, including 'oxidative stress response', 'axon guidance mediated by netrin' and 'salvage pyrimidine deoxyribonucleotides'. Cytoscape software was used to further analyze the hsa-miR-1246 regulated genes (Fig. 5B).

In addition, as presented in Fig. 5C, the KEGG pathway clustering based on the hsa-miR-892b regulated genes were involved in 11 pathways, including 'Wnt signaling pathway', 'cadherin signaling pathway' and 'notch signaling 


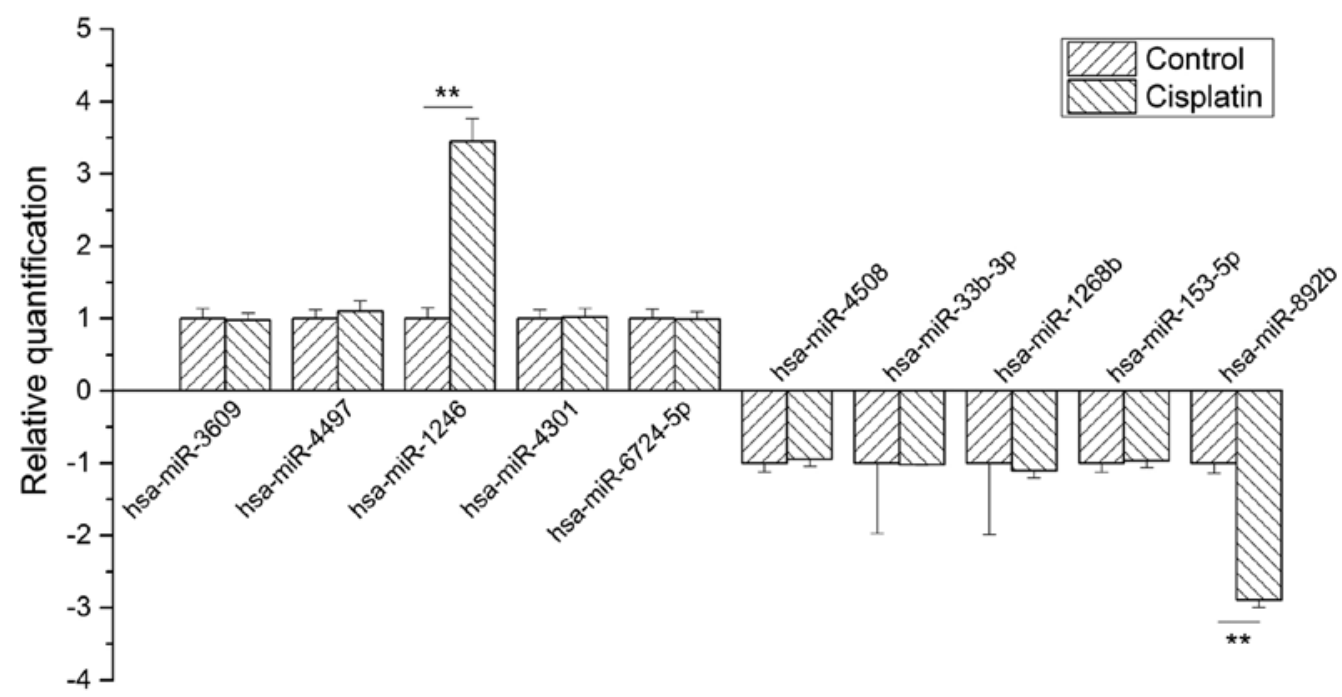

Figure 3. Expression levels of the top five significantly upregulated and top five significantly downregulated miRNAs as identified by reverse transcription-quantitative polymerase chain reaction analysis. The expression level of hsa-miR-1246 was significantly increased, and that of hsa-miR-892b was significantly decreased in NCI-N87 cells treated with cisplatin compared with control cells. This was consistent with the results of microarray analysis. ${ }^{* *} \mathrm{P}<0.01$ vs. control. miR, microRNA.

A

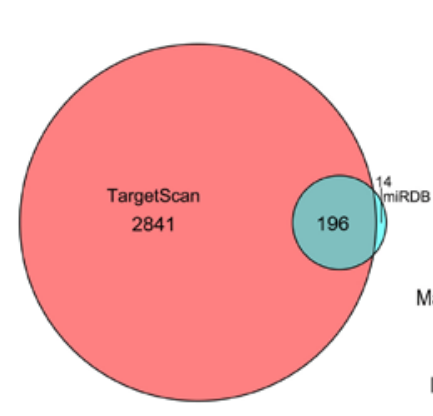

B

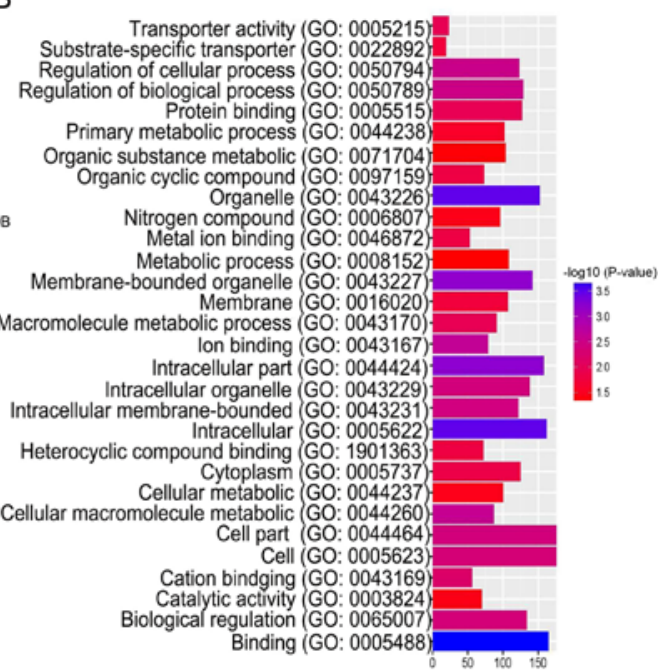

D

C

Reculation of cellular process (GO: 0050794) Regulation of biological process (GO: 0050789) Protein binding (GO: 0005515)
Primary metabolic process (GO: 0044238) Plasma membrance (GO: 0005886) Organic substance metabolic (GO: 0071704), Organic compound binding (GO: 0097159) Organelle (GO: 0043226)
Nitrogen compound metabolic (GO: 0006807)
Multicellular organismal process (GO: 0032501).

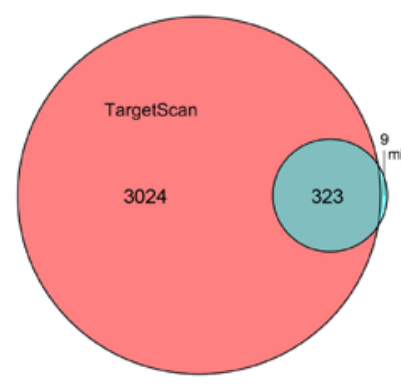
Multicellular organismal process (GO: 0032501) Metal ion binding (GO: 0046872)
Metabolilc process (GO: 0008152) Macromolecule metabolic process (GO: 0043170 lon binding (GO: 0043167) Intracellular organelle (GO: 0043229 ) Intracellular (GO: 0005622) Heterocyclic compound binding (GO: 1901363). Cellular metabolic process (GO: 0044237)
Cell periphery (GO: 0071944) Cell part (GO: 0044464)Cell (GO: 0005623) Cation binding (GO: 0043169), Catalytic activity (GO: 0003824)
Biological regulation (GO: 0065007)

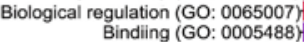
Anon binding (GO: 0043168) ]

Figure 4. Investigation of hsa-miR-1246- and hsa-miR-892b-regulated genes by Venn diagram analysis and GO clustering. (A) The intersecting genes of hsa-miR-1246-regulated genes by Venn diagram analysis. (B) The intersecting genes of hsa-miR-1246-regulated genes analysis by GO clustering. (C) The intersecting genes of hsa-miR-892b-regulated genes by Venn diagram analysis. (D) The intersecting genes of hsa-miR-892b-regulated genes analysis by GO clustering. miR, microRNA; GO, Gene Ontology; miRDB, microRNA Target Prediction and Functional Study Database. 
A

Salvage pyrimidine deoxyribonucleotides

(P02774)

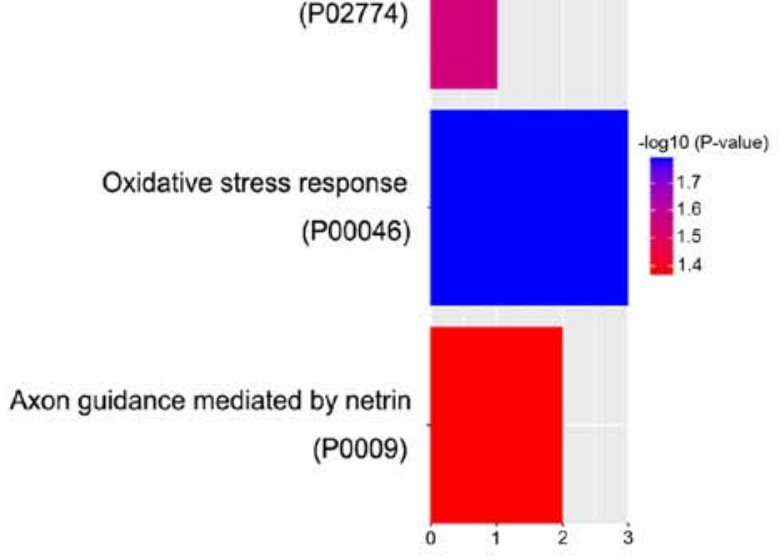

C

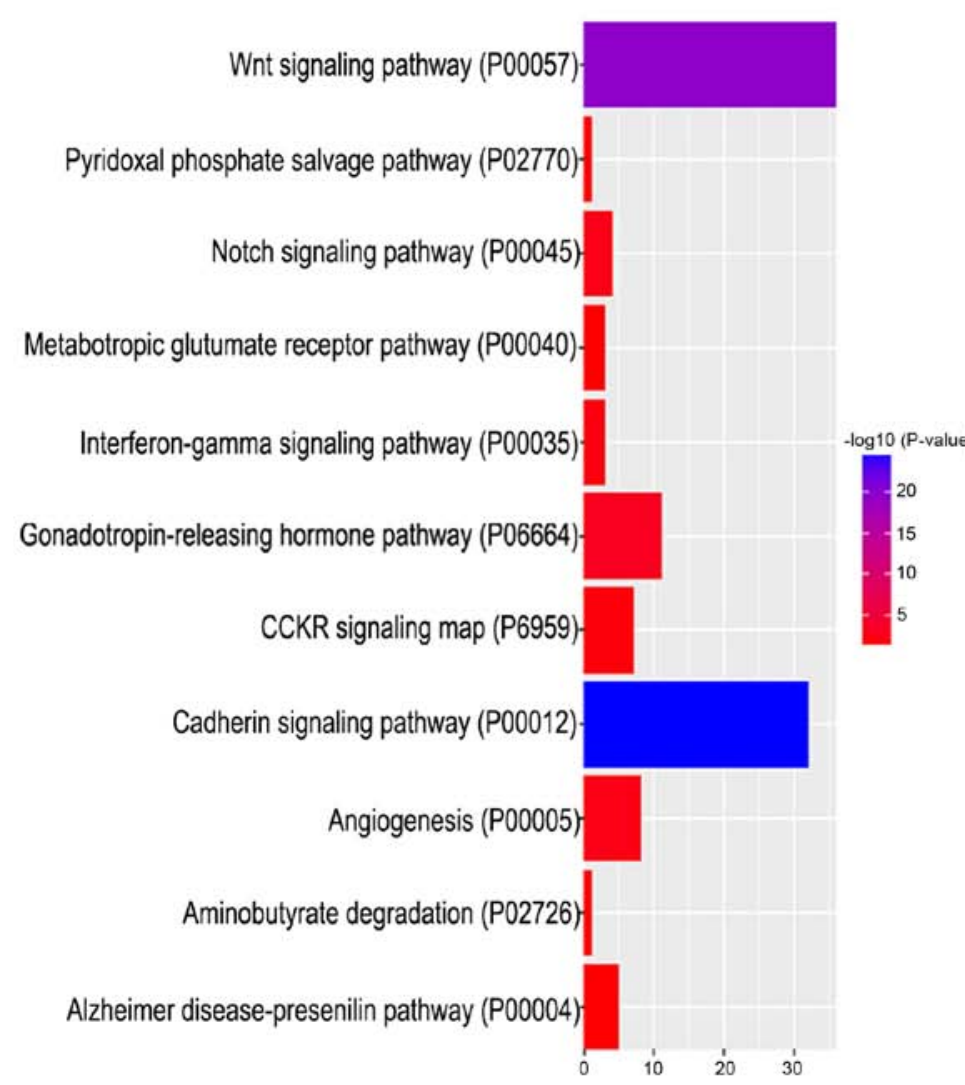

D
B

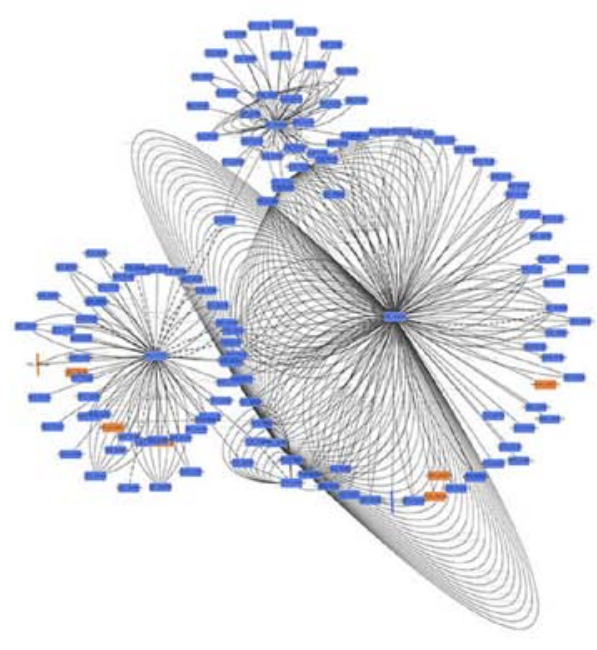

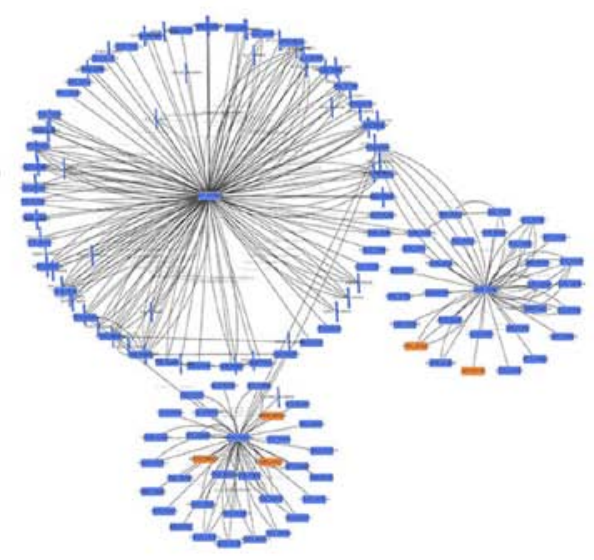

Figure 5. KEGG pathway analysis of hsa-miR-1246 and hsa-miR-892b-regulated genes by histogram and cross-talk network. (A) The histogram exhibited the KEGG pathway of the hsa-miR-1246 regulated genes. (B) The cross-talk network analysis of the hsa-miR-1246 regulated genes. (C) The histogram exhibited the KEGG pathway of the hsa-miR-892b-regulated genes. (D) The cross-talk network analysis of the hsa-miR-892b-regulated genes. KEGG, Kyoto Encyclopedia of Genes and Genomes; miR, microRNA.

pathway'. Cytoscape software was used to further analyze the hsa-miR-892b regulated genes (Fig. 5D).

\section{Discussion}

In the current study, the human gastric cancer cell line NCI-N87 was cultured with the $15 \mu \mathrm{g} / \mathrm{ml}$ of cisplatin, and high-throughput sequencing combined with RT-qPCR was used to detect cisplatin-regulated genes. Finally, miRNAs that were positively and negatively associated with cisplatin treatment were analyzed statistically, and confirmed 33 positively- and 16 negatively-associated miRNAs. The top five significantly upregulated and top five significant down-regulation miRNAs were selected to further verify the results 
obtained from the microarray analysis. It was revealed that the expression levels of hsa-miR-1246 and hsa-miR-892b were consistent with microarray analysis. GO and KEGG pathway clustering of cisplatin-regulated miRNAs was performed, and it was demonstrated that the two miRNAs were involved in several BPs and signaling pathways. Therefore, cisplatin may exert an important anticancer effect in gastric cancer by affecting biological processes and signal pathways; however further investigation is required.

Gastric cancer is the second leading cause of cancer-associated mortalities in the world, and has a poor prognosis as the majority of patients are diagnosed at an advanced stage (18). Although there have been improvements in the early diagnosis and treatment of gastric cancer, the prognosis of patients with gastric cancer is generally not favorable $(32,33)$. One of the main reasons for this observation is primary or secondary drug resistance during chemotherapy (34). A number of mechanisms have been proposed to explain the phenomenon of drug resistance in cancer cells (34). Previous studies revealed that tumor drug-resistance mechanisms included oncogene activation, anti-oncogene inactivation, reduced intracellular drug concentration, drug target molecular changes, metabolism detoxification, enhanced DNA damage repair function and inhibition of tumor cell apoptosis (28-30). Therefore, elucidating the mechanisms underlying the occurrence and development of gastric cancer drug resistance in order to reverse this process may improve treatment outcomes in gastric cancer. Cisplatin is one of the most commonly used drugs in the treatment of advanced cancer, including gastric cancer (35). Despite the high sensitivity of patients with gastric cancer to cisplatin at initial administration, a substantial number of patients develop drug resistance, which is one of the major causes of treatment failure (36). Cisplatin-based combination chemotherapy is the most effective treatment for metastatic gastric cancer (37). However, chemoresistance remains an obstacle for the effective treatment of the disease (38).

miRNAs are small non-coding RNAs that function as endogenous silencers of various target genes (39). Mature miRNAs bind to the 3'untranslated regions of target mRNA, leading to the silencing of mRNA (39). miRNAs regulate biological functions, including cell proliferation, differentiation and apoptosis. A number of studies have demonstrated that miRNAs are involved in the occurrence, development, diagnosis and treatment of cancer $(40,41)$. Certain miRNAs, such as miR-34a, are downregulated in various types of cancer and act as tumor suppressors $(42,43)$. Conversely, miRNAs such as miR-155 are reportedly overexpressed in various types of cancer, and act as oncogenes (44). Changes in miRNA expression contribute to the initiation and progression of cancer $(45,46)$. The association between miRNAs and tumors suggests that miRNAs may be altered in patients with gastric cancer $(47,48)$. A previous study demonstrated that miR-218 increased chemosensitivity to cisplatin in vitro and in vivo by inducing apoptosis (18). The current study revealed that cisplatin upregulated hsa-miR-1246 and downregulated hsa-miR-892b. Few studies have investigated the role of hsa-miR-1246 in cancer; however, it has been reported that miR-1246 is associated with the apoptosis and migration of cancer cells (49). Furthermore, miR-1246 has a p53-responsive element in its promoter region; p53 was determined to induced hsa-miR-1246 expression (49). In non-small cell lung cancer, hsa-miR-1246 plays an important role in the invasion of cancer cells by promoting proliferation, and sphere and colony formation (50); however, its role in gastric cancer has not been reported. The role of hsa-miR-892b in tumor progression, particularly in gastric cancer, remains unclear.

The results obtained in the current study suggested that hsa-miR-1246 and hsa-miR-892b may play important roles in the progression of gastric cancer via certain BPs and signaling pathways; however, future studies are required to confirm this. The high incidence of false positives obtained in the current study may be associated with the small sample size and the use of only one cell line. A larger sample size and other cell lines are required for future work.

The current study investigated cisplatin-regulated genes in NCI-N87 cells. The results obtained may provide a theoretical basis for a novel approach to further study the association between miRNA and cisplatin resistance in gastric cancers, as well as its possible mechanism. Importantly, differential miRNA expression patterns may provide a solid basis for further functional studies to identify potential oncogenic or tumor suppressor miRNAs in gastric cancer during cisplatin resistance. Of note, as our study was conducted in a preliminary matter, further investigation is required to determine the direct target genes of cisplatin and the mechanism underlying the effects of cisplatin on gastric cancer.

\section{Acknowledgements}

Not applicable.

\section{Funding}

No funding was received.

\section{Availability of data and materials}

The datasets used and/or analyzed during the current study are available from the corresponding author on reasonable request.

\section{Authors' contributions}

$\mathrm{CY}$ and $\mathrm{XM}$ conceived and designed the experiments. CY, XZ, $\mathrm{HX}$ and HL performed the experiments. CY, XZ, MG and KY contributed to the acquisition and analysis of data. CY and $\mathrm{XM}$ wrote, reviewed and edited the manuscript.

\section{Ethics approval and consent to participate}

Not applicable.

\section{Patient consent for publication}

Not applicable.

\section{Competing interests}

The authors declare that they have no competing interests. 


\section{References}

1. Podratz JL, Lee H, Knorr P, Koehler S, Forsythe S, Lambrecht K, Arias S, Schmidt K, Steinhoff G, Yudintsev G, et al: Cisplatin induces mitochondrial deficits in Drosophila larval segmental nerve. Neurobiol Dis 97: 60-69, 2017.

2. Bobylev I, Joshi AR, Barham M, Neiss WF and Lehmann HC: Depletion of mitofusin-2 causes mitochondrial damage in cisplatin-induced neuropathy. Mol Neurobiol 55: 1227-1235, 2018.

3. Shkalim-Zemer V, Ash S, Toledano H, Kollender Y, Issakov J, Yaniv I and Cohen IJ: Highly effective reduced toxicity dose-intensive pilot protocol for non-metastatic limb osteogenic sarcoma (SCOS 89. Cancer Chemother Pharmacol 76: 909-916, 2015.

4. Ma G, Cai H, Gao L, Wang M and Wang H: sCLU regulates cisplatin chemosensitivity of lung cancer cells in vivo. World J Surg Oncol 13: 80, 2015.

5. Li P, Yang X, Cheng Y, Zhang X, Yang C, Deng X, Li P, Tao J, Yang H, Wei J, et al: MicroRNA-218 increases the sensitivity of bladder cancer to cisplatin by targeting Glut1. Cell Physiol Biochem 41: 921-932, 2017.

6. Allen JC, Kirschner A, Scarpato KR and Morgans AK: Current management of refractory germ cell tumors and future directions. Curr Oncol Rep 19: 8, 2017.

7. Pellat A, Wislez M, Svrcek M, Hammel P, Afchain P and Andre T: Therapeutic management of poorly differentiated neuroendocrine lung tumors and neuroendocrine carcinomas of the digestive system. Bull Cancer 103: 880-895, 2016.

8. Durusu IZ, Husnugil HH, Atas H, Biber A, Gerekci S, Gulec EA and Özen C: Anti-cancer effect of clofazimine as a single agent and in combination with cisplatin on U266 multiple myeloma cell line. Leuk Res 55: 33-40, 2017.

9. Starkov AK, Zamay TN, Savchenko AA, Ingevatkin EV, Titova NM, Kolovskaya OS, Luzan NA, Silkin PP and Kuznetsova SA: Antitumor effect of arabinogalactan and platinum complex. Dok1 Biochem Biophys 467: 92-94, 2016.

10. Jamesdaniel S, Rathinam R and Neumann WL: Targeting nitrative stress for attenuating cisplatin-induced downregulation of cochlear LIM domain only 4 and ototoxicity. Redox Biol 10: 257-265, 2016

11. Leo M, Schmitt LI, Erkel M, Melnikova M, Thomale J and Hagenacker T: Cisplatin-induced neuropathic pain is mediated by upregulation of $\mathrm{N}$-type voltage-gated calcium channels in dorsal root ganglion neurons. Exp Neurol 288: 62-74, 2017.

12. Lee J, Kim HH, Ro SM and Yang JH: Capecitabine and cisplatin (XP) combination systemic chemotherapy in heavily pre-treated HER2 negative metastatic breast cancer. PLoS One 12: e0171605, 2017.

13. Vergaro V, Papadia P, Petrini P, Fanizzi FP, De Pascali SA, Baldassarre F, Pastorino L and Ciccarella G: Nanostructured polysaccharidic microcapsules for intracellular release of cisplatin. Int J Biol Macromol 99: 187-195, 2017.

14. Cherniawsky H, Merchant N, Sawyer M and Ho M: A case report of posterior reversible encephalopathy syndrome in a patient receiving gemcitabine and cisplatin. Medicine (Baltimore) 96: e5850, 2017.

15. Hassan SM, Khalaf MM, Sadek SA and Abo-Youssef AM: Protective effects of apigenin and myricetin against cisplatin-induced nephrotoxicity in mice. Pharm Biol 55: 766-774, 2017.

16. Karafakioglu YS, Bozkurt MF, Hazman O and Fidan AF: Efficacy of safranal to cisplatin-induced nephrotoxicity. Biochem J 474: 1195-1203, 2017.

17. Sokolova $\mathrm{O}$ and Naumann M: NF- $\kappa \mathrm{B}$ signaling in gastric cancer. Toxins 9: pii: E119, 2017.

18. Chen XZ, Chen H, Castro FA, Hu JK and Brenner H: Epstein-Barr virus infection and gastric cancer: A systematic review. Medicine (Baltimore) 94: e792, 2015.

19. Wang C, Zhang J, Cai M, Zhu Z, Gu W, Yu Y and Zhang X: DBGC: A database of human gastric cancer. PLoS One 10: e0142591, 2015.

20. Long ZW, Yu HM, Wang YN, Liu D, Chen YZ, Zhao YX and Bai L: Association of IL-17 polymorphisms with gastric cancer risk in Asian populations. World J Gastroenterol 21: 5707-5718, 2015.

21. Wu HH,Lin WC and Tsai KW: Advances in molecular biomarkers for gastric cancer: miRNAs as emerging novel cancer markers. Expert Rev Mol Med 16: e1, 2014.

22. Huang D, Duan H, Huang H, Tong X, Han Y, Ru G, Qu L, Shou C and Zhao Z: Cisplatin resistance in gastric cancer cells is associated with HER2 upregulation-induced epithelial-mesenchymal transition. Sci Rep 6: 20502, 2016.
23. Xu W, Wang S, Chen Q, Zhang Y, Ni P, Wu X, Zhang J, Qiang F, Li A, Roe OD, et al: TXNL1-XRCC1 pathway regulates cisplatin-induced cell death and contributes to resistance in human gastric cancer. Cell Death Dis 5: e1055, 2014.

24. Korobkina EA, Knyazeva MS, Kil YV, Titov SE and Malek AV: Comparative analysis of RT-qPCR based methodologies for microRNA detection. Klin Lab Diagn 63: 722-728, 2018.

25. Kumar D, Das PK and Sarmah BK: Reference gene validation for normalization of RT-qPCR assay associated with germination and survival of rice under hypoxic condition. J Appl Genet 59: 419-430, 2018.

26. Zou J, Dong X, Li Y, Tong S, Wang J, Liao M and Huang G: Deep sequencing identification of differentially expressed miRNAs in the spinal cord of resiniferatoxin-treated rats in response to electroacupuncture. Neurotox Res: May 23, 2019 (Epub ahead of print).

27. Ashburner M, Ball CA, Blake JA, Botstein D, Butler H, Cherry JM, Davis AP, Dolinski K, Dwight SS, Eppig JT, et al: Gene ontology: Tool for the unification of biology. The Gene Ontology Consortium. Nat Genet 25: 25-29, 2000.

28. The Gene Ontology Consortium: The Gene Ontology Resource: 20 years and still GOing strong. Nucleic Acids Res 47: D330-D338, 2019.

29. Kanehisa M, Sato Y, Furumichi M, Morishima K and Tanabe M: New approach for understanding genome variations in KEGG. Nucleic Acids Res 47: D590-D595, 2019.

30. Agarwal V, Bell GW, Nam JW and Bartel DP: Predicting effective microRNA target sites in mammalian mRNAs. Elife: 4: e05005, 2015.

31. Wong N and Wang X: miRDB: An online resource for microRNA target prediction and functional annotations. Nucleic Acids Res 43 (Database Issue): D146-D152, 2015.

32. Fock KM: Review article: The epidemiology and prevention of gastric cancer. Aliment Pharmacol Ther 40: 250-260, 2014.

33. Kamata S, Kishimoto T, Kobayashi S, Miyazaki M and Ishikura H: Possible involvement of persistent activity of the mammalian target of rapamycin pathway in the cisplatin resistance of AFP-producing gastric cancer cells. Cancer Biol Ther 6: 1036-1043, 2007.

34. He J, Qi H, Chen F and Cao C: MicroRNA-25 contributes to cisplatin resistance in gastric cancer cells by inhibiting forkhead box O3a. Oncol Lett 14: 6097-6102, 2017.

35. Li X, Liang J, Liu YX, Wang Y, Yang XH, Luan BH, Zhang GL, Du J and Wu XH: miR-149 reverses cisplatin resistance of gastric cancer SGC7901/DDP cells by targeting FoxM1. Die Pharmazie 71: 640-643, 2016.

36. Silberman H: Perioperative adjunctive treatment in the management of operable gastric cancer. J Surg Oncol 90: 174-187, 2005

37. Ajani JA, Buyse M, Lichinitser M, Gorbunova V, Bodoky G, Douillard JY, Cascinu S, Heinemann V, Zaucha R, Carrato A, et al: Combination of cisplatin/S-1 in the treatment of patients with advanced gastric or gastroesophageal adenocarcinoma: Results of noninferiority and safety analyses compared with cisplatin/5-fluorouracil in the First-Line Advanced Gastric Cancer Study. Eur J Cancer 49: 3616-3624, 2013.

38. Park SC and Chun HJ: Chemotherapy for advanced gastric cancer: Review and update of current practices. Gut Liver 7: 385-393, 2013.

39. Bartel DP: MicroRNAs: Genomics, biogenesis, mechanism, and function. Cell 116: 281-297, 2004.

40. Xia S, Guo J, Li J, Zhou L and Zhao Y: Application of miRNAs in the occurrence and early diagnosis of pancreatic cancer. Zhonghua Wai Ke Za Zhi 52: 198-201, 2014 (In Chinese).

41. Wang CM, Yang XL, Liu MH, Cheng BH, Chen J and Bai B: High-throughput sequencing analysis of differentially expressed miRNAs and target genes in ischemia/reperfusion injury and apelin-13 neuroprotection. Neural Regen Res 13: 265-271, 2018.

42. Sun X, Huang T, Liu Z, Sun M and Luo S: LncRNA SNHG7 contributes to tumorigenesis and progression in breast cancer by interacting with miR-34a through EMT initiation and the Notch-1 pathway. Eur J Pharmacol 856: 172407, 2019.

43. Zhu M, Zheng Z, Huang J, Ma X, Huang C, Wu R, Li X, Liang Z, Deng F, Wu J, et al: Modulation of miR-34a in curcumin-induced antiproliferation of prostate cancer cells. J Cell Biochem: May 1, 2019 (Epub ahead of print).

44. Fukuda K, Arigami T, Yanagita S, Matsushita D, Okubo K, Kijima T, Uenosono Y, Ishigami S and Natsugoe S: A case of advanced gastric cancer showing pathological complete response after neoadjuvant chemotherapy with S-1 and oxaliplatin. Gan To Kagaku Ryoho 46: 471-473, 2019. 
45. Kalapanida D, Zagouri F, Gazouli M, Zografos E, Dimitrakakis C, Marinopoulos S, Giannos A, Sergentanis TN, Kastritis E, Terpos E, et al: Evaluation of pre-mir-34a rs72631823 single nucleotide polymorphism in triple negative breast cancer: A case-control study. Oncotarget 9: 36906-36913, 2018.

46. Orosz E, Kiss I, Gyongyi Z and Varjas T: Expression of circulating miR-155, miR-21, miR-221, miR-30a, miR-34a and miR-29a: Comparison of colonic and rectal cancer. In Vivo 32: 1333-1337, 2018

47. Anauate AC, Leal MF, Wisnieski F, Santos LC, Gigek CO, Chen ES, Calcagno DQ, Assumpcao PP, Demachki S, Arasaki CH, et al: Analysis of 8q24.21 miRNA cluster expression and copy number variation in gastric cancer. Future Med Chem: May 29, 2019 (Epub ahead of print).
48. Zhang Z, Dong Y, Hua J, Xue H, Hu J, Jiang T, Shi L and Du J: A five-miRNA signature predicts survival in gastric cancer using bioinformatics analysis. Gene 699: 125-134, 2019.

49. Hibino S, Saito Y, Muramatsu T, Otani A, Kasai Y, Kimura M and Saito H: Inhibitors of enhancer of zeste homolog 2 (EZH2) activate tumor-suppressor microRNAs in human cancer cells. Oncogenesis 3: e104, 2014.

50. Kim G, An HJ, Lee MJ, Song JY, Jeong JY, Lee JH and Jeong HC Hsa-miR-1246 and hsa-miR-1290 are associated with stemness and invasiveness of non-small cell lung cancer. Lung Cancer 91: 15-22, 2016. 